\title{
Prevalencia de alteraciones músculoesqueléticas en niños escolares con obesidad en atención primaria
}

\section{Prevalence of musculoskeletal disorders in school age children with obesity in primary care}

DOI: $10.46932 / \mathrm{sfjdv2n3-067}$

Received in: May 1st, 2021

Accepted in: Jun 30th, 2021

\section{González Pérez Brian}

Higher academic background: Médico Familiar., Máster en Ciencias Médicas., Máster en Innovación y Tecnología Educativa., Profesor Investigador.

Current Institution: Servicio de Atención Familiar. Unidad Médica Familiar 38, Tampico Tamaulipas, Instituto Mexicano del Seguro Social, México. Facultad de Medicina "Dr. Alberto Romo Caballero"

Universidad Autónoma de Tamaulipas, Tampico, Tamaulipas, México.

Full address: Calle Argentina y Canada 107 Col. Infonavit C.P. 89310, Tampico, Tamaulipas.

Email: brian.gonzalez.perez@gmail.com

\section{Ricardo Salas Flores}

Higher academic background: Endocrinólogo Pediatra., Master en Ciencias Médicas., Profesor Investigador.

Current Institution: Coordinación de Educación en Salud e Investigación. Hospital General Regional No. 6, Madero, Tamaulipas, Instituto Mexicano del Seguro Social, México. Facultad de Medicina "Dr.

Alberto Romo Caballero" Universidad Autónoma de Tamaulipas, Tampico, Tamaulipas, México.

Full address: Blvd. Adolfo López Mateos S/N, Col. Las Conchitas, Madero Tamaulipas.

\section{Liliana Aurora Carrillo Aguiar}

Higher academic background: Médico Familiar., Master en Ciencias Médicas., Profesor Titular de la Residencia en Medicina Familiar.

Current Institution: Unidad Médica Familiar 77, Madero Tamaulipas, Instituto Mexicano del Seguro Social, México.

Full address: Blvd. Adolfo López Mateos S/N, Col. Las Conchitas, Madero Tamaulipas.

\section{Eduardo Mata Zaleta}

Higher academic background: Médico Familiar.

Current Institution: Unidad Médica Familiar 77, Madero Tamaulipas, Instituto Mexicano del Seguro Social, México.

Full address: Blvd. Adolfo López Mateos S/N, Col. Las Conchitas, Madero Tamaulipas.

\section{Roandy Gaspar Hernández Carranco}

Higher academic background: Doctorado en Ciencias de la Enfermería., Profesor Investigador. Current Institution: Facultad de Enfermería de Tampico. Universidad Autónoma de Tamaulipas,

Tampico, Tamaulipas, México.

Full address: Centro Universitario Sur, Cto. Interior S/N CP 89337, Tampico, Tamaulipas

\section{Anaya Maldonado Aidaly}

Higher academic background: Estudiante de Medicina. Programa Delfín, XXVI Verano de la Investigación Científica y Tecnológica del Pacífico. 
Current Institution: Facultad de Medicina “Dr. Alberto Romo Caballero” Universidad Autónoma de

Tamaulipas, Tampico, Tamaulipas, México.

Full address: Centro Universitario Sur, Cto. Interior S/N CP 89337, Tampico, Tamaulipas

\author{
Alonso Ruiz María Esther \\ Higher academic background: Estudiante de Medicina. Programa Delfín, XXVI Verano de la \\ Investigación Científica y Tecnológica del Pacífico. \\ Current Institution: Facultad de Medicina "Dr. Alberto Romo Caballero" Universidad Autónoma de \\ Tamaulipas, Tampico, Tamaulipas, México. \\ Full address: Centro Universitario Sur, Cto. Interior S/N CP 89337, Tampico, Tamaulipas
}

\title{
Manriquez Vazquez Jorge Alfredo
}

Higher academic background: Estudiante de Medicina. Programa Delfín, XXVI Verano de la Investigación Científica y Tecnológica del Pacífico.

Current Institution: Facultad de Medicina "Dr. Alberto Romo Caballero" Universidad Autónoma de

Tamaulipas, Tampico, Tamaulipas, México.

Full address: Centro Universitario Sur, Cto. Interior S/N CP 89337, Tampico, Tamaulipas

\begin{abstract}
RESUMEN
INTRODUCCIÓN: En las últimas cuatro décadas la obesidad se ha convertido en una epidemia que ha afectado la salud de la población mundial. En la infancia es donde se propicia principalmente el desarrollo musculoesquelético, por lo cual una buena adquisición de la postura erguida y de las habilidades motoras básicas es fundamental. A pesar de la búsqueda de la consecuencia de la obesidad infantil en el desarrollo y función del sistema musculoesquelético ha recibido relativamente poca atención. Realizar la investigación propuesta permitirá: identificar las complicaciones y realizar una revisión sistemática acerca de las repercusiones de la obesidad infantil a nivel musculoesquelético, permitirá desarrollar acciones de diagnóstico y tratamiento oportunos, para evitar el desarrollo de todas las patologías y complicaciones mencionadas
\end{abstract}

OBJETIVO: Determinar la prevalencia de alteraciones musculoesqueléticas en niños escolares con obesidad de la unidad de medicina familiar no 77 de Ciudad Madero Tamaulipas.

MÉTODOS: Estudio clínico no experimental, prospectivo, transversal, que incluyó niños entre 5 y 12 años con obesidad que acudan a consulta a la Unidad de Medicina Familiar 77 seleccionados mediante un muestreo no probabilístico por conveniencia, que aceptaron participar en el estudio, previa aplicación del consentimiento y asentimiento informado. Se les pidió a los padres contesten un cuestionario referente a la ficha de identificación de su hijo, en otra sección contestar si ha tenido la presencia de dolor en extremidades inferiores, antecedentes de lesiones a nivel de la articulación de la cadera, piernas o tobillos, posteriormente se realizó somatometría y valoración de los pies con un podoscopio para confirmar o descartar pie plano. Se realizó análisis descriptivo, las variables cuantitativas se expresaron como medias y desviación estándar, y las nominales como frecuencias y porcentajes. El proyecto cumplió con la declaración de Helsinki, Informe Belmont, Ley General de Salud, Reglamento de la Ley General de Salud en materia de investigación para la salud, considerándose como investigación con riesgo mínimo.

RESULTADOS: Los hallazgos de este estudio se reportan con un análisis de los parámetros cuantitativos de la población estudiada, Del total de casos, la edad mínima fue de 5 y el máximo de 12 años, con una media de 8.5, mediana de 8 , y moda de 7 , con una desviación estándar de 2.14. El porcentaje por grupo de edad fue: $76.5 \%$ entre 5 y 7 años y de $23.3 \%$ entre 8 y 12 años de edad. La distribución por sexo fue: $5 \%$ mujeres (15) y 95\% hombres (285). Del total de la población estudiada, el $5 \%$ tenía pie plano. La presencia de dolor en extremidades inferiores, la refirió el $2.17 \%$ de los casos y el total de estos, en la extremidad derecha. Ninguno de los casos refirió antecedente de fractura de tobillos. Genu Valgo se encontró en el $18.69 \%$ de los casos incluidos en el estudio. No se reportaron Genu Varo. Respecto a los 
antecedentes de lesiones a nivel de la articulación de la cadera, piernas o tobillos, solo se reportaron del total de los casos un $2.3 \%$ con antecedente de esguince de tobillo.

CONCLUSIONES: Los hallazgos de esta investigación sugieren que la población infantil con dicha patología tiene una mayor incidencia y prevalencia de alteraciones musculoesqueléticas. Por ello la prevención de la obesidad desde las primeras etapas de vida es clave para prevenir dichas repercusiones y evitar un impacto negativo durante las etapas de desarrollo del infante. La visión acerca de la repercusión de la obesidad a nivel musculoesquelético se amplió y se evidenció la necesidad de crear nuevas líneas de investigación que permitan una visión más concreta de todas las repercusiones en esta etapa del desarrollo

Palabras claves: Obesidad, alteraciones musculoesqueléticas, niños, escolares, sobrepeso, enfermedades crónicas

\begin{abstract}
INTRODUCTION: In the last four decades obesity has become an epidemic that has affected the health of the world population. It is mainly in childhood that musculoskeletal development takes place, so a good acquisition of upright posture and basic motor skills is essential. Despite the research, the consequence of childhood obesity on the development and function of the musculoskeletal system has received relatively little attention. Performing the proposed research will allow: to identify the complications and perform a systematic review about the repercussions of childhood obesity at the musculoskeletal level, will allow to develop timely diagnostic and treatment actions, to avoid the development of all the mentioned pathologies and complications.

OBJETIVE: Determine the prevalence of musculoskeletal disorders in school children with obesity of the family medicine unit no 77 of Ciudad Madero Tamaulipas

METHODS: Non-experimental, prospective, cross-sectional clinical study, which will include children between 5 and 12 years old with obesity who come to the Family Medicine Unit 77 selected through a non-probabilistic convenience sampling, who agree to participate in the study after applying their consent and informed assent. Parents will be asked to answer a questionnaire regarding their child's identification card, in another section to answer if they have had pain in the lower extremities, history of injuries at the level of the hip, leg or ankle joint, Subsequently, somatometry and evaluation of the feet with a podoscope will be performed to confirm or rule out flat feet. Descriptive analysis will be carried out, quantitative variables will be expressed as means and standard deviation, and the nominal ones as frequencies and percentages. The project complies with the Declaration of Helsinki, Belmont Report, General Health Law, Regulation of the General Health Law on research for health, considering it as research with minimal risk. RESULTS: The findings of this study are reported with an analysis of the quantitative parameters of the population studied, of the total number of cases, the minimum age was 5 and the maximum was 12 years, with a mean of 8.5 , median of 8 , and mode of 7 , with a standard deviation of 2.14 . The percentage by age group was $76.5 \%$ between 5 and 7 years of age and $23.3 \%$ between 8 and 12 years of age. The distribution by sex was: $5 \%$ females (15) and $95 \%$ males (285). Of the total population studied, $5 \%$ had flat feet. The presence of pain in the lower extremities was reported in $2.17 \%$ of the cases and the total of these, in the right extremity. None of the cases reported a history of ankle fracture. Genu Valgus was found in $18.69 \%$ of the cases included in the study. Genu varum was not reported. Regarding the history of injuries at the level of the hip joint, legs, or ankles, only $2.3 \%$ of the total cases reported a history of ankle sprain.

CONCLUSIONS: The findings of this research suggest that children with this pathology have a higher incidence and prevalence of musculoskeletal alterations. Therefore, the prevention of obesity from the early stages of life is key to prevent such repercussions and avoid a negative impact during the developmental stages of the infant. The vision of the repercussions of obesity at the musculoskeletal level was broadened and the need to create new lines of research that allow a more concrete vision of all the repercussions at this stage of development became evident.
\end{abstract}

Key words: Obesity, musculoskeletal alterations, children, schoolchildren, overweight, chronic diseases 


\section{INTRODUCCIÓN}

En las últimas cuatro décadas la obesidad se ha convertido en una epidemia que ha afectado la salud de la población mundial. La OMS define al sobrepeso y obesidad como una acumulación anormal de grasa que se debe a un desequilibrio energético entre las calorías consumidas y gastadas. La obesidad es considerada una enfermedad crónico-degenerativa de origen multifactorial donde se incluyen tanto causas genéticas como ambientales, además de ser factor de impacto en la vida social del individuo, afectar a nivel psicológico y de desarrollar comorbilidades las cuales son enfermedades crónicas no transmisibles que son totalmente prevenibles, entre ellas, diabetes, hipertensión, afectación cardiovascular, alteraciones musculoesqueléticas entre otras (Pérez y López, 2018)

La obesidad infantil constituye uno de los principales problemas que enfrenta la salud pública (Diaz, 2017), se estima que a nivel mundial 40-50 millones de niños en edad escolar se clasifican como obesos y que 200 millones padecen sobrepeso (Machado et al., 2018). En México, la encuesta nacional de salud y nutrición (ENSANUT) 2018 indica que la prevalencia de sobrepeso y obesidad en los niños de 5-11 años en el 2016 es de $35.6 \%$ siendo $18.1 \%$ con sobrepeso y $17.5 \%$ con obesidad.

En la infancia es donde se propicia principalmente el desarrollo musculoesquelético, por lo cual una buena adquisición de la postura erguida y de las habilidades motoras básicas es fundamental. La obesidad se considera como uno de los factores externos que generan cambios en la postura corporal de los niños, sin embargo, no se tienen pruebas sobre el efecto que tiene la obesidad infantil con la postura. (García, 2020)

Los niveles altos de masa grasa que se presentan en la obesidad pueden resultar dañinos tanto en el desarrollo óseo como en complicaciones metabólicas, todo esto relacionado al aumento del tejido adiposo. (Dimitri, 2018)

Zhou et al. (2018) demostraron que una carga alta de adipocitos en el cuerpo puede generar un estrés de carga, además de dañar severamente lo que son los sistemas osteomuscular y articular, causando que se creen deformidades articulares y procesos inflamatorios y degenerativos, causando que el niño tenga una función física baja y dolor.

La obesidad es considera también un factor de riesgo para la espondilolistesis epifisaria femoral unilateral o bilateral y varo tibial, causando que los infantes no puedan tener una vida activa y tengan un estilo de vida sedentario, causando que el bajar de peso sea difícil. (Villarrasa, 2019)

En el estudio que dirigió Ferro (2018), se expone que "los niños con obesidad y sobrepeso tenían más probabilidades de sufrir fracturas en las extremidades inferiores” (p.7). Esto sugiere que hay una 
mayor incidencia de fracturas, teniendo también el IMC alto como factor para asociarse a fracturas severas.

El niño con sobrepeso y obesidad es más propenso a un peor desarrollo motor y problemas de ortopedia así lo afirma Sailaja, V., Amarnath, K. y Narsing, B. (2018). Cuando hay una disminución del arco longitudinal interno del pie se le denomina pie plano que es asociado posteriormente a fracturas mal consolidadas o luxaciones que dejan deformidades permanentes y enfermedades inflamatorias (Córdova, 2015). En el Genu valgo, los miembros inferiores toman un aspecto de " $x$ " debido a que las rodillas se desvían hacia adentro de la línea media, en infantes con sobrepeso se estima que existe una progresión acelerada del mal alineamiento por efecto de carga en su valgo fisiológico (Ibáñez, 2017). Por otro lado, en el Genu varo, se torna un aspecto de paréntesis porque las rodillas se desvían hacia afuera de la línea media todas estas patologías asociadas al sobrepeso y obesidad. (Ramos de Ascanio, C. 2020).

México está entre los primeros lugares en obesidad infantil a nivel mundial, problema que se presenta más a menudo en los estados del norte y en comunidades urbanas. (UNICEF, 2015). A pesar de la búsqueda la consecuencia de la obesidad infantil en el desarrollo y función del sistema musculoesquelético ha recibido relativamente poca atención. Realizar la investigación propuesta permitirá: identificar las complicaciones y realizar una revisión sistemática acerca de las repercusiones de la obesidad infantil a nivel musculoesquelético, permitirá desarrollar acciones de diagnóstico y tratamiento oportunos, para evitar el desarrollo de todas las patologías y complicaciones mencionadas. El objetivo en la realización de esta investigación fue determinar la prevalencia de los problemas musculoesqueléticos en niños escolares entre los 5 y los 12 años con sobrepeso y obesidad que acudieron a consulta a la Unidad de Medicina Familiar 77 del Instituto Mexicano del Seguro Social.

\section{MÉTODOS:}

Estudio clínico no experimental, prospectivo, transversal, en niños de ambos sexos entre 5 y 12 años de edad con obesidad que acudieron a consulta a la Unidad de Medicina Familiar 77 durante el periodo comprendido de enero a diciembre 2019. Se seleccionaron mediante un muestreo no probabilístico por conveniencia, que aceptaron participar en el estudio previa aplicación del consentimiento y asentimiento informado. Se les solicitó a los padres contestaran un cuestionario referente a la ficha de identificación de su hijo, en otra sección contestar si ha tenido la presencia de dolor en extremidades inferiores, antecedentes de lesiones a nivel de la articulación de la cadera, piernas o tobillos, posteriormente se realizó somatometría y valoración de los pies con un podoscopio para confirmar o descartar pie plano. Se realizó análisis descriptivo, las variables cuantitativas se expresarán como medias y desviación estándar, y las nominales como frecuencias y porcentajes. El proyecto cumple con la 
declaración de Helsinki, Informe Belmont, Ley General de Salud, Reglamento de la Ley General de Salud en materia de investigación para la salud, considerándose como investigación con riesgo mínimo. Fue aprobado por el Comité Local de Investigación en Salud 2801 del Instituto Mexicano del Seguro Social.

\section{RESULTADOS}

Los hallazgos de la investigación se reportan con un análisis de los parámetros cuantitativos de la población estudiada. Todos sugieren que la población infantil con sobrepeso y obesidad se relaciona con una mayor incidencia de alteraciones musculoesqueléticas. La prevalencia encontrada fue de $27 \%$ (Gráfica 1 y 2).

Gráfica 1. Prevalencia de las alteraciones musculoesqueléticas en el total de la población infantil con obesidad incluida en la investigación

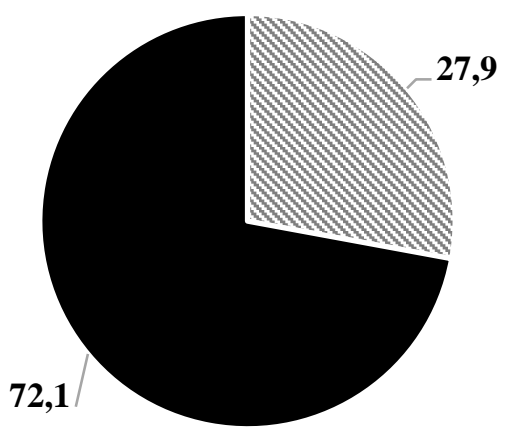

s Con Alteraciones ME

- Sin Alteraciones ME
Gráfica 2. Prevalencia. Porcentaje de las alteraciones musculoesqueléticas más frecuentes en niños con sobrepeso y obesidad en la población estudiada.

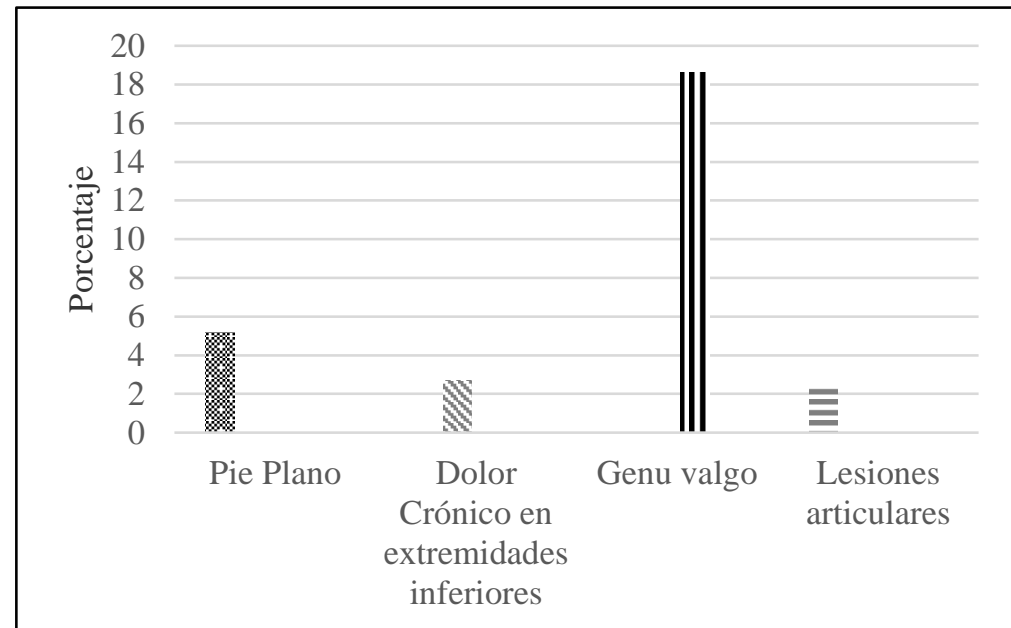

Del total de casos (300), la distribución por edad fue: edad mínima de 5 años y máxima de 12, con una media de 8.5, mediana de 8 y moda de 7. Desviación estándar de 2.14 (Gráfica 3). 
Gráfica 3. Distribución de casos por edad

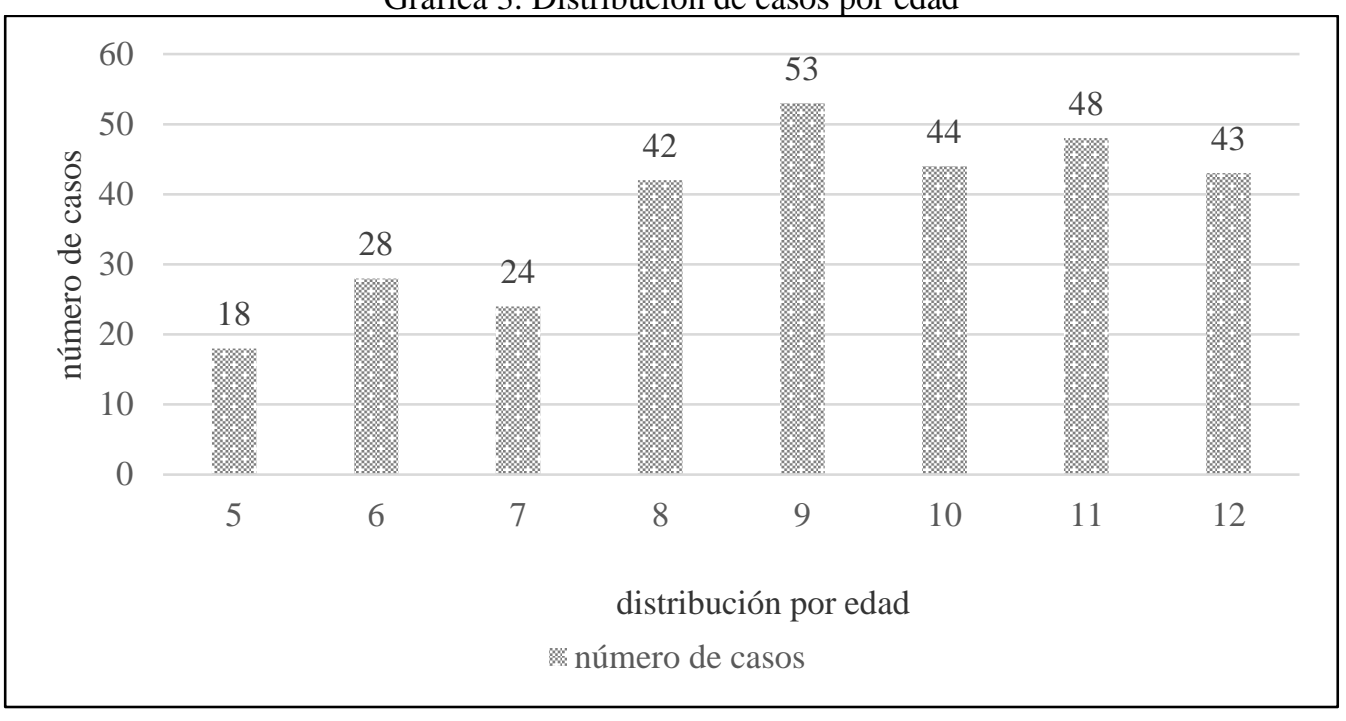

La distribución por grupo de edad fue: el 76.5\% de la población corresponde al grupo de edad entre y 7 años; el $23.3 \%$ al grupo entre 8 y 12 años. El mayor porcentaje de alteraciones musculoesqueléticas relacionadas a sobrepeso y obesidad fue en el rango de edad de 5 a 7 años (Gráfica 4).

Gráfica 4. Distribución por edad. Porcentaje de problemas musculoesqueleticos respecto a los grupos de edad.

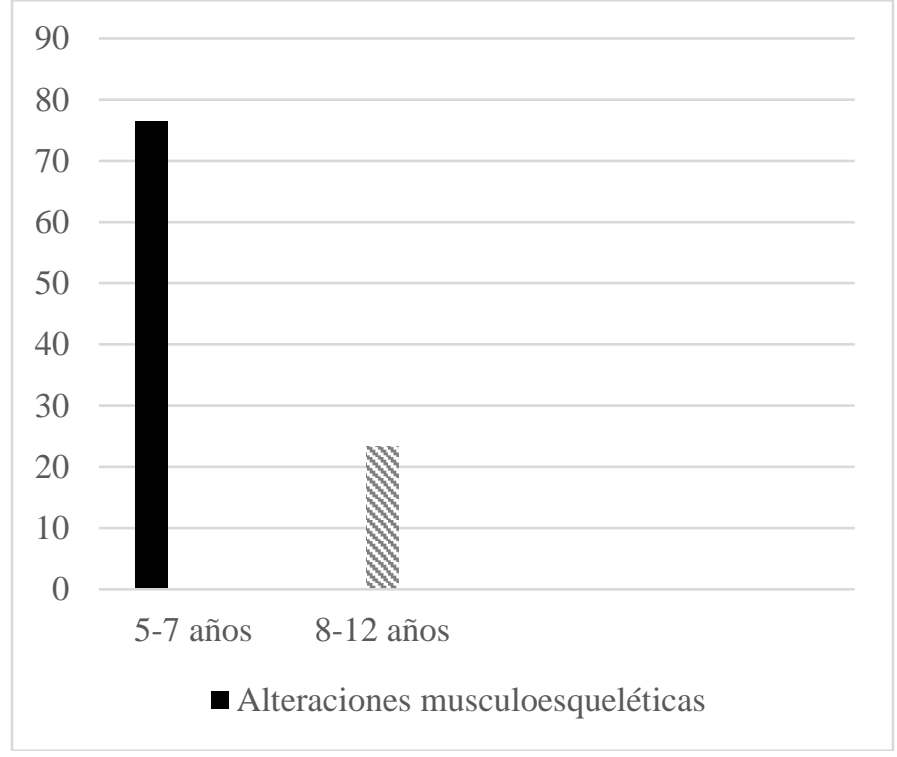

Respecto al porcentaje de niños y niñas, 15 casos, es decir el 5\% de nuestra población fueron niñas y 285 casos, es decir el $95 \%$ correspondió a niños. (Gráfica 5,6). 
Gráfica 5. Distribución de alteraciones musculoesqueléticas por sexo.

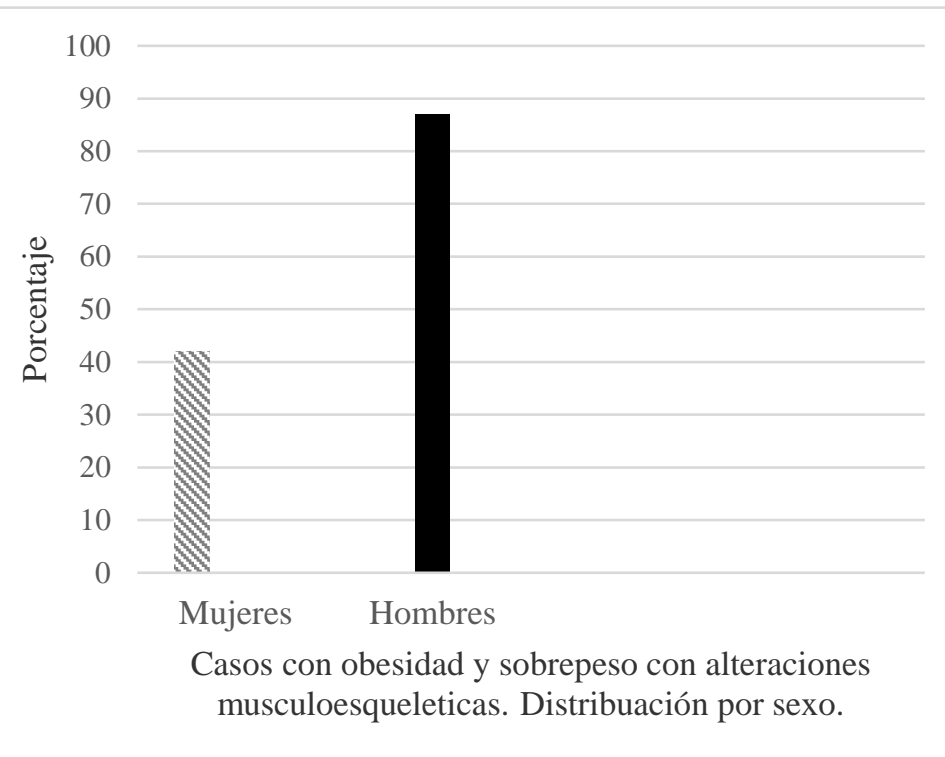

Gráfica 6. Distribución por sexo de casos con obesidad y sobrepeso.

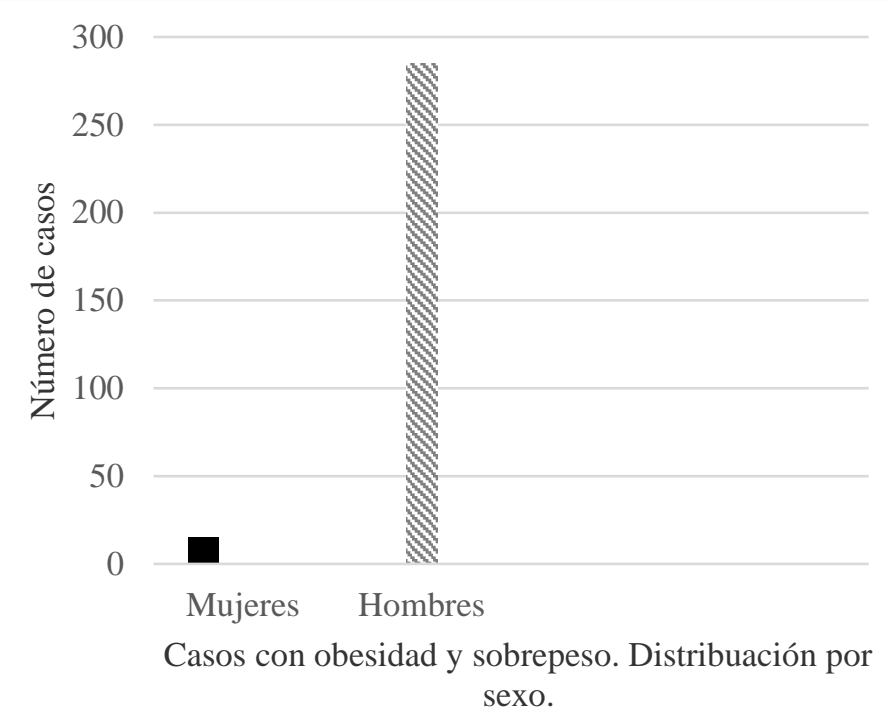

Alteraciones musculoesqueléticas específicas: PIE PLANO. Esta alteración se encontró en el 5\% de casos totales. (Gráfica 7)

Gráfica 7. En la población estudiada, solo el 5.2\% presentaba pie plano.

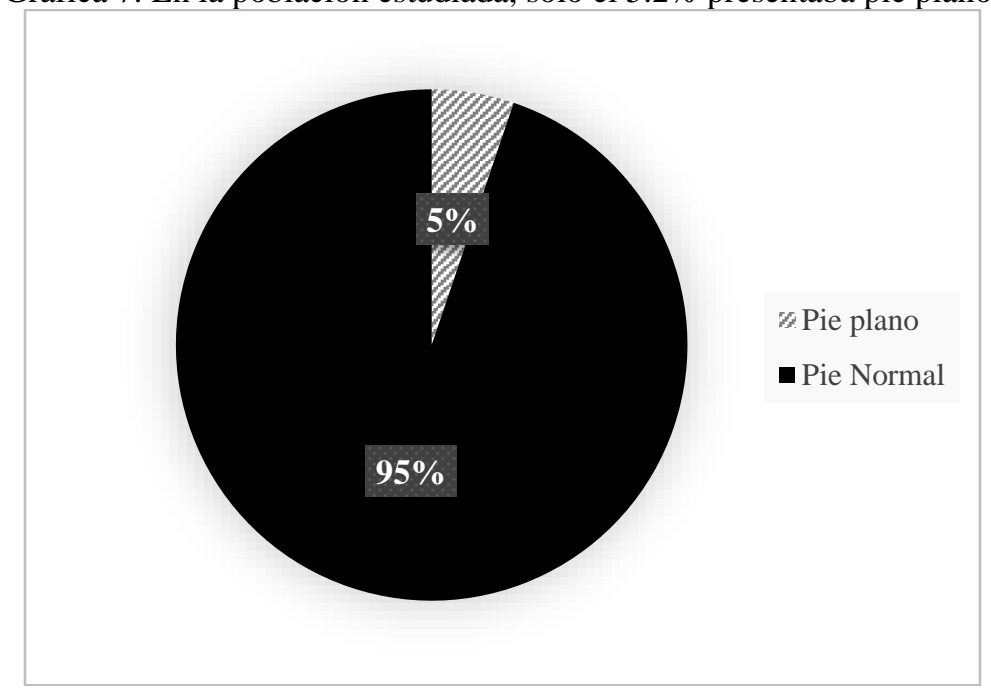

Entre la población que se encontró con PIE PLANO, el porcentaje de distribución por grupo de edad y sexo fue: en el grupo de edad de 5 a 7 años, el $72 \%$ fueron hombres y el $28 \%$ mujeres; en el grupo de 8 a 12 años, el 9\% fueron hombres y el 91\% mujeres. (Gráfica 8) 
Gráfica 8. Porcentaje de casos de PIE PLANO distribuidos grupo de edad y sexo.

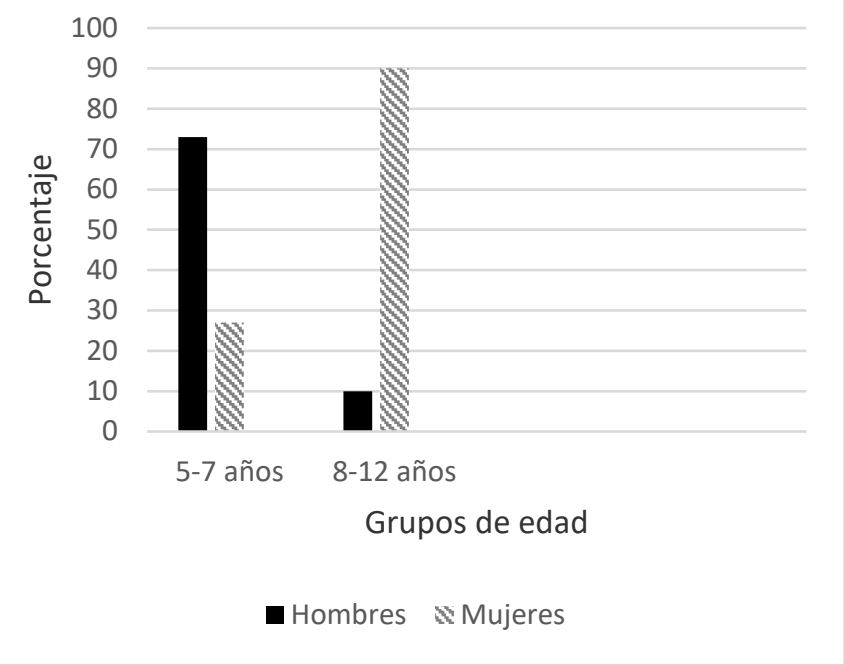

DOLOR CRONICO EN EXTREMIDADES INFERIORES: del total de casos, el dolor se presentó en $2.17 \%$ y el 100\% de estos casos fue en el lado derecho. EI GENU VALGO se encontró en el $18.69 \%$ de los casos. No se encontraron casos con GENU VARO. (Grafica 9 y 10)

Gráfica 9. De la población estudiada, solo el $2.17 \%$ refirió la presencia de dolor en las últimas 2 semanas anterior a la encuesta, sin reportar EVA específico, y en todos los casos con dolor positivo, éste era en la extremidad inferior derecha

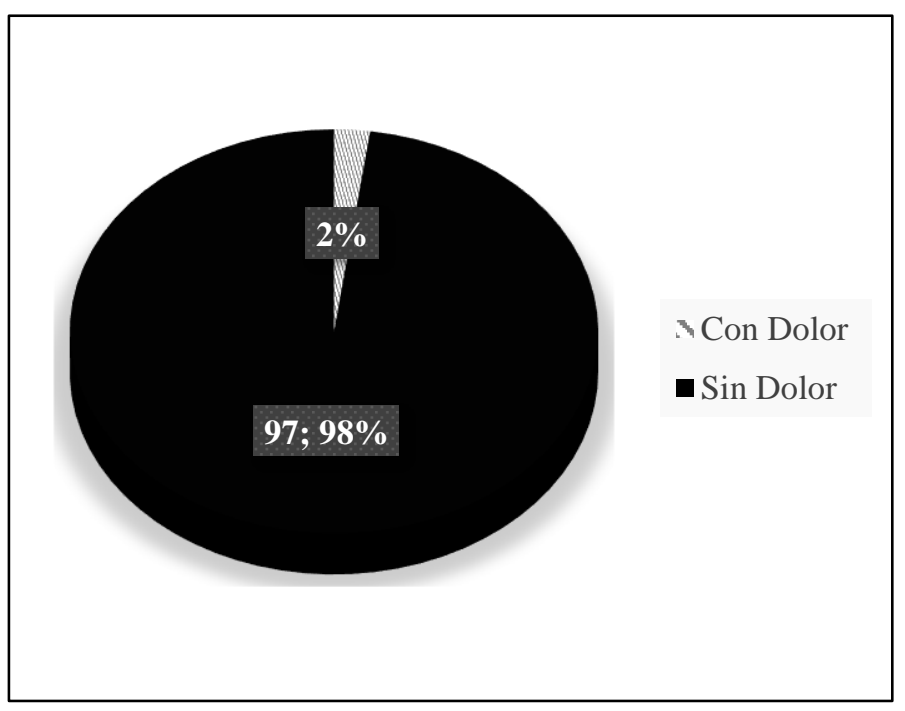

Gráfica 10. Porcentaje de población, con dolor crónico en extremidades inferiores al momento de la revisión. Distribución de porcentaje de casos en hombres y mujeres.

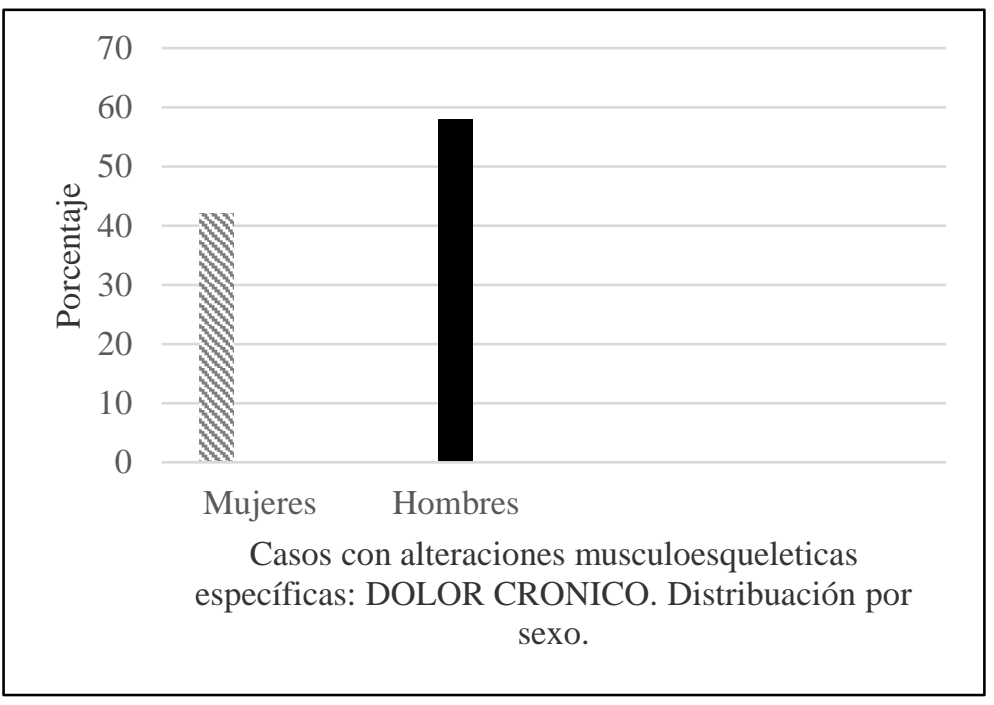

Ninguno de los casos tuvo antecedente de fracturas en extremidades inferiores. Respecto a los antecedentes o hallazgos de lesiones a nivel articular de cadera, piernas o tobillos, del total de casos se encontraron estas alteraciones en un $2.3 \%$. 


\section{DISCUSIÓN}

En todos los estudios que se revisaron incluyendo este, se ha observado que las alteraciones musculoesqueléticas han sido relacionadas con la obesidad y que poseen un impacto en edades tempranas, se encontró que la obesidad afecta a las articulaciones de las extremidades inferiores, como las rodillas, tobillos y pies; las cuales se encuentran expuestas a cargas mecánicas mayores, generando micro lesiones que se asocian a un dolor musculoesquelético.

El rango de edad en el que más se encontraron alteraciones musculoesqueléticas fue en el de 5 a 7 años, teniendo un porcentaje mayor al de 8 a 12 años.

Además, los dos grupos de edad se encontraron por encima de las tallas y pesos normales, teniendo un porcentaje aproximado del $90 \%$ de cada uno de sus grupos, algo que nos hace confirmar sobre la presencia de problemas de obesidad y sobrepeso en nuestra población blanco, aunque, como se menciona en un artículo de Molina-Gracia (2019), no se tiene una clasificación por categorías como se tienen en los casos de adultos con obesidad, es más aceptada el uso de la medición del IMC.

Una de las alteraciones con mayor número de casos entre la población fue del genu valgo, el cual se encontró en el $18.69 \%$ de los casos, lo cual se confirma que es frecuente en niños con sobrepeso y obesidad. Un IMC más alto de lo normal, se ha relacionado en la población infantil con el problema de valgo tibial, pero se ha encontrado también que, debido a la maduración esquelética, puede predecir más la gravedad del genu valgo. (Walker, 2019)

El genu varo no se encontró en ninguno de los 300 casos estudiados, pero numerosos estudios han confirmado que existe una predisposición más en jóvenes que practican fútbol excesivamente, como lo menciona Ginesin (2018) en su estudio, en donde se encontró el genu varo en la mayoría de las jóvenes futbolistas a comparación de los que no practican el deporte.

Entre la población estudiada se encontró que el $5 \%$ de los casos totales presentan la alteración musculoesquelética de pie plano. En el grupo de edad de 5 a 7 años se localizó esta alteración en su mayoría en los hombres en contraste en el grupo de edad de 8 a 12 años predominó el número de casos en las mujeres. Se puede comparar con el estudio realizado por Pourghasame et al donde el sexo masculino tuvo mayor prevalencia en la presencia de pie plano, aunque comenta que se suele esperar una mayor incidencia de pie plano en el sexo femenino debido a que presentan huesos más débiles y el tipo de calzado que utilizan puede ser perjudicial para el desarrollo del arco longitudinal plantar. (Pourghasame et al, 2016)

En el $2.17 \%$ de la población se presentó dolor crónico en la extremidad inferior derecha tal como se muestra en otros estudios, la obesidad posee un efecto negativo sobre la salud musculoesquelética de los niños. 
De todos los casos, en ninguno se encontró antecedentes de fracturas en las extremidades inferiores, esto se puede comparar a que estudios recientes han mencionado que en niños obesos se encuentran niveles altos de minerales en los huesos. (Fintini, 2020) Aun así, se encontraron lesiones en lo que es nivel articular, teniendo un total de $2.3 \%$.

La obesidad infantil sigue siendo un problema para la sociedad que trae consigo distintas repercusiones que aún no están del todo estudiadas, se trata de una enfermedad crónica no transmisible que es totalmente prevenible, para su tratamiento es necesario el trabajo de un equipo multidisciplinar basado en tres pilares: un cambio en los hábitos alimenticios, un aumento en la actividad física y el apoyo personal y familiar.

\section{CONCLUSIONES}

La obesidad es una enfermedad que actúa como factor de riesgo de comorbilidad que es potencialmente evitable. Los hallazgos de esta investigación sugieren que la población infantil con dicha patología tiene una mayor incidencia y prevalencia de alteraciones musculoesqueléticas. Por ello la prevención de la obesidad desde las primeras etapas de vida es clave para prevenir dichas repercusiones y evitar un impacto negativo durante las etapas de desarrollo del infante. Derivado de lo encontrado en la literatura y de los resultados del estudio realizado, la visión acerca de la repercusión de la obesidad a nivel musculoesquelético se amplió y se evidencio la necesidad de crear nuevas líneas de investigación que permitan una visión más concreta de todas las repercusiones en esta etapa del desarrollo. Siendo conscientes de esta asociación y su impacto en la salud infantil, es necesario investigar más, mediante la propuesta de nuevas hipótesis y líneas de investigación futuras. 


\section{REFERENCIAS}

Aranceta-Bartrina, J., \& Pérez-Rodrigo, C. (2018). Childhood Obesity: An Unresolved Issue. Revista española de cardiología (English ed.), 71(11), 888-891. https://doi.org/10.1016/j.rec.2018.05.04

Blanco, M., Veiga, O. L., Sepúlveda, A. R., Izquierdo-Gomez, R., Román, F. J., López, S., \& Rojo, M. (2020). Ambiente familiar, actividad física y sedentarismo en preadolescentes con obesidad infantil: estudio ANOBAS de casos-controles [Family environment, physical activity and sedentarism in preadolescents with childhood obesity: ANOBAS case-control study]. Atención primaria, 52(4), 250257. https://doi.org/10.1016/j.aprim.2018.05.013

Córdova Constantino, Jorge Lisandro, \& Regino Juan, Julio César, \& de la Cruz Gil, Elías, \& López Morales, Cynthia, \& Pimentel Domínguez, Brenda Cristina (2015). Prevalencia de defectos posturales de miembros inferiores en pacientes de 2 meses a 14 años de edad del Centro de Rehabilitación y Educación Especial de Tabasco. Salud en Tabasco, Julio de 2021]. ISSN: 1405-2091. Disponible en: https://www.redalyc.org/articulo.oa?id=48745738003

21 (2-3), 55-61. [Fecha de Consulta 23 de

Dimitri P. (2018). Fat and bone in children - where are we now?. Annals of pediatric endocrinology \& metabolism, 23(2), 62-69. https://doi.org/10.6065/apem.2018.23.2.62

Díaz Martín J. J. (2017). Obesidad infantil: ¿prevención o tratamiento? [Childhood obesity: Prevention or treatment?]. Anales de pediatria (Barcelona, Spain : 2003), 86(4), 173- 175. https://doi.org/10.1016/j.anpedi.2016.12.004

Ferro, V., Mosca, A., Crea, F., Mesturino, M. A., Olita, C., Vania, A., Reale, A., Nobili, V., \& Raucci, U. (2018). The relationship between body mass index and children's presentations to a tertiary pediatric emergency department. Italian journal of pediatrics, 44(1), 38. https://doi.org/10.1186/s13052-018-0476-y

Fintini, D., Cianfarani, S., Cofini, M., Andreoletti, A., Ubertini, G. M., Cappa, M., \& Manco, M. (2020). The Bones of Children With Obesity. Frontiers in endocrinology, 11, 200. https://doi.org/10.3389/fendo.2020.00200

García, P. M. (2020). Biomechanics of childhood obesity: implications for the musculoskeletal system and role of physical exercise (Doctoral dissertation, Universidad de Granada). https://digibug.ugr.es/handle/10481/60171

Ginesin, E., Norman, D., \& Peskin, B. (2018). Knee Alignment and Its Significance: Is It Really Different in Various Population Groups?. The Israel Medical Association journal : IMAJ, 20(2), https://www.ima.org.il/MedicineIMAJ/viewarticle.aspx?year=2018\&month=02\&page =109

Ibáñez A. Manifestaciones ortopédicas de la obesidad en niños y adolescentes. Rev. Actuali. Clinic. Meds. Vol. 1. Num 1, Enero-Junio (2017). ISSN 0719-8620, pp 33-41.

Medina, C., Jáuregui, A., Campos-Nonato, I., \& Barquera, S. (2018). Prevalencia y tendencias de actividad física en niños y adolescentes: resultados de Ensanut 2012 y Ensanut MC $2016 . \quad$ Salud Pública de México, 60(3, may-jun), 263. https://doi.org/10.21149/8819 
Molina-Garcia, P., Migueles, J. H., Cadenas-Sanchez, C., Esteban-Cornejo, I., Mora-Gonzalez, J., Rodriguez-Ayllon, M., Plaza-Florido, A., Vanrenterghem, J., \& Ortega, F. B. (2019). A systematic review on biomechanical characteristics of walking in children and adolescents with overweight/obesity: Possible implications for the development of musculoskeletal disorders. Obesity reviews : an official journal of the International Association for the Study of Obesity, 20(7), 10331044. https://doi.org/10.1111/obr.12848

Obesidad y sobrepeso. (2021, 9 junio). Obesidad y sobrepeso. https://www.who.int/es/newsroom/fact-sheets/detail/obesity-and-overweight

Pourghasem, M., Kamali, N., Farsi, M., \& Soltanpour, N. (2016). Prevalence of flatfoot among school students and its relationship with BMI. Acta orthopaedica et traumatologica turcica, 50(5), 554557. https://doi.org/10.1016/j.aott.2016.03.002

Pérez-Herrera, A., \& Cruz-López, M. (2019). Situación actual de la obesidad infantil en México [Childhood obesity: current situation in Mexico]. Nutricion hospitalaria, 36(2), 463-469. https://doi.org/10.20960/nh.2116

Ramos de Ascanio, C. (2020). Estudio observaciones retrospectivo sobre el tratamiento de genu valgo en la infancia en un hospital universitario de tercer nivel. Universidad de La Laguna. http://riull.ull.es/xmlui/handle/915/20367

Sailaja, V., Amarnath, K. y Narsing, B. (2018). Un estudio sobre la prevalencia de la obesidad infantil en escuelas privadas de la población de la ciudad de Kurnool. Revista nacional de fisiología, farmacia y farmacología, 8 (9), 1302.

Sobrepeso/obesidad en niños en edad escolar y sus factores de riesgo. (2018). Archivos de Pediatría del Uruguay. Published. https://doi.org/10.31134/ap.89.s1.2

UNICEF. (2015). Encuesta Nacional de Niños, Niñas y Mujeres. 2015. https://www.unicef.org/mexico/informes/encuesta-nacional-de-ni\%C3\%B1os- ni\%C3\%B1asy-mujeres-2015

Villarrasa Sapiña, I. (2019). Influencia de la obesidad infantil sobre el control postural y la marcha. (Tesis Doctoral, Universidad de Valencia). https://roderic.uv.es/handle/10550/70567

Walker, J. L., Hosseinzadeh, P., White, H., Murr, K., Milbrandt, T. A., Talwalkar, V. J., $\quad$ Iwinski, H., \& Muchow, R. (2019). Idiopathic Genu Valgum and Its Association With Obesity in Children and Adolescents. Journal of pediatric orthopedics, 39(7), 347-352. https://doi.org/10.1097/BPO.0000000000000971

Zhou, M., Chen, J., Wang, D., Zhu, C., Wang, Y., \& Chen, W. (2018). Combined effects of reproductive and hormone factors and obesity on the prevalence of knee osteoarthritis and knee pain among middle-aged or older Chinese women: a cross-sectional study. BMC public health, 18(1), 1192. https://doi.org/10.1186/s12889-018-6114-1 